\title{
Trombocitose como Fator Prognóstico no Câncer Colorretal
}

\author{
Trombocitosis as a Prognostic Factor in Colorectal Cancer
}

\author{
RENATO DEARAÚJO BONARDI ${ }^{1}$, KATIA GRACIOSA $^{2}$, EDUARDO FELIPPE MELCHIORETTO $^{3}$, LIZANDRO FRAINER \\ FURLANI $^{2}$, MARIA CRISTINASARTOR $^{4}$, ANTÔNIO BALDIN JR $^{5}$
}

\begin{abstract}
${ }^{1}$ Prof. Adjunto Doutor do Depto. de Cirurgia e Chefe dos Serviços de Cirurgia Geral e Coloproctologia da UFPR; ${ }^{2}$ Médicos Residentes da Unidade de Coloproctologia do Hospital de Clínicas da UFPR; ${ }^{3}$ Médico Residente do Serviço de Cirurgia Geral do Hospital de Clínicas da UFPR; ${ }^{4}$ Doutora em Cirurgia do Aparelho Digestivo pela USP e Médica da Unidade de Coloproctologia do Hospital de Clínicas da UFPR; ${ }^{5}$ Mestre em Microbiologia, Parasitologia e Patologia pela UFPR e Médico da Unidade de Coloproctologia da UFPR.
\end{abstract}

\begin{abstract}
BONARDI RA; GRACIOSA K; MELCHIORETTO EF; FURLANI LF; SARTOR MC; BALDIN JR A. Trombocitose como Fator Prognóstico no Câncer Colorretal. Rev bras Coloproct, 2010;30(2): 128-133.

RESUMO: Objetivos - Investigar o significado prognóstico da trombocitose nos pacientes com câncer colorretal. Método - Tratase de estudo retrospectivo, com análise de $\mathbf{2 4 3}$ prontuários de pacientes submetidos a operações por câncer colorretal. Foram comparados os dados do estadiamento, recidiva tumoral e óbitos por câncer com a ocorrência de trombocitose no pré-operatório. O grupo controle foi composto de 50 pacientes submetidos à herniorrafia. A média da contagem de plaquetas no pré-operatório destes pacientes foi utilizada para dividir os pacientes em dois grupos: grupo 1, pacientes com contagem de plaquetas abaixo dessa média e grupo 2, pacientes com contagem de plaquetas acima dessa média. Resultados - A média da contagem plaquetária foi 317000/il entre os pacientes com câncer e de 267000 entre os pacientes do grupo controle. A prevalência da trombocitose no câncer colorretal foi $32,1 \%$. Dentre os óbitos por câncer, 56,7\% ocorreram em pacientes com trombocitose e 32\% em pacientes com

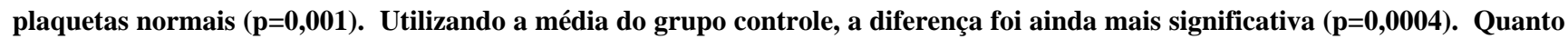
à recidiva tumoral, $40 \%$ dos pacientes do grupo 2 tiveram recidiva e 17,9\% do grupo $1(\mathrm{p}=0,003)$. Com relação ao estadiamento $\mathrm{T}$, no grupo 1, 14,1\% eram T1 e 8,4\% T4. No grupo 2, 2,2\% eram T1 e 19,5\% T4 (p=0.0005). Metástases à distância foram encontradas em 9,4\% dos pacientes do grupo 1 contra $21,8 \%$ do grupo 2 (p=0.02). No que diz respeito ao estadiamento TNM, no grupo 1, 24,6\% eram estadio 1 e $11 \%$ estadio 4. No grupo 2, 9,6\% eram estadio 1 e 22,8\% estadio 4 (p=0,02). Conclusão - A contagem de plaquetas no pré-operatório parece ser útil em identificar pacientes com prognóstico desfavorável.
\end{abstract}

Descritores: Trombocitose; câncer colorretal; prognóstico; plaquetas.

\section{INTRODUÇÃO}

O câncer colorretal é a segunda causa de morte por câncer em todo mundo ${ }^{1}$. Existem inúmeros fatores prognósticos, clínicos e patológicos, identificados até o momento. Dentre eles, os mais importantes são o envolvimento linfonodal e a presença de metástases à distância ${ }^{2}$. Entretanto, a recidiva ocorre em cerca de 20 a $30 \%$ dos pacientes que não apresentam estes fatores no momento da cirurgia $^{3}$. A identificação no pré-operatório de ou- tros fatores associados a um risco aumentado de recorrência pode auxiliar no acompanhamento e tratamento desses pacientes ${ }^{4}$.

A associação de trombocitose com doenças malignas é conhecida há mais de 100 anos $^{5}$. Aproximadamente um terço de todos os paciente com câncer apresentam trombocitose no momento do diagnóstico, e $40 \%$ de todos os pacientes com trombocitose apresentam uma doença maligna ${ }^{6}$.

Recentemente, a trombocitose foi descrita em pacientes com câncer do pulmão, estômago, vias

Trabalho realizado no Serviço de Coloproctologia da Disciplina de Cirurgia Geral do Hospital de Clínicas da UFPR - Curitiba - PR - Brasil.

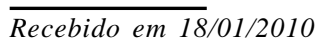

Aceito para publicação em 27/04/2010 
urinárias e ginecológicos ${ }^{7-12}$. Entretanto, seu valor prognóstico ainda é controverso na literatura.

Existem vários estudos mostrando que a trombocitose é fator de mau prognóstico em vários tipos de câncer. Porém, dados mostrando seu impacto no câncer colorretal são escassos ${ }^{13}$.

Nesse estudo foi investigado a trombocitose no pré-operatorio e seu significado no prognóstico de pacientes com câncer colorretal.

\section{MÉTODO}

Trata-se de estudo retrospectivo, com análise de prontuários de 243 pacientes submetidos a operações por câncer colorretal no Hospital de Clínicas da Universidade Federal do Paraná (HC-UFPR) no período de janeiro de 2003 a dezembro de 2008.

Só foram incluídos no estudo pacientes que apresentassem adenocarcinoma colorretal comprovado através do anatomo-patológico, registro da contagem plaquetária pré-operatória e seguimento ambulatorial no HC-UFPR. Foram excluídos pacientes com diagnóstico de doença inflamatória intestinal, por estes já apresentarem trombocitose devido à doença.

Foram comparados os dados do estadiamento, recidiva tumoral e óbitos por câncer com a ocorrência de trombocitose no pré-operatório. Foi considerado trombocitose a contagem plaquetária acima de 350.000/ microlitro. Dezoito pacientes não apresentavam hemograma com o número absoluto de plaquetas e foram incluídos somente na análise qualitativa (com ou sem trombocitose).

Utilizou-se, na maioria das vezes, o ánatomopatológico. Nos casos em que não houve ressecção do tumor por completo foi considerado o estadiamento cirúrgico.

Foi selecionado um grupo controle, composto de 50 pacientes submetidos à herniorrafia no Serviço de Cirurgia Geral do HC-UFPR. Este grupo foi escolhido, pois faz parte da avaliação pré-operatória de rotina destas operações, a realização de hemograma completo com contagem plaquetária, não interferindo na rotina pré-estabelecida para que os dados necessários à pesquisa sejam obtidos.

A média da contagem de plaquetas no préoperatório destes pacientes foi utilizada para dividir os pacientes com câncer colorretal em dois grupos: grupo 1 - pacientes com contagem de plaquetas abaixo dessa média;

grupo 2 - pacientes com contagem de plaquetas acima dessa média.

A análise estatística foi realizada utilizando o programa Epiinfo 6.0. Foi considerado estatisticamente significativo um $\mathrm{p}<0.05$.

\section{RESULTADOS}

Duzentos e quarenta e três pacientes com média de idade de 59 anos (variação de 25 a 99 anos) foram incluídos no estudo. Desses, $56.4 \%$ eram do sexo masculino e $43.6 \%$ do sexo feminino. O reto foi a localização mais comum dos tumores, perfazendo $51 \%$ dos casos. (Tabela 1)

A tabela 2 mostra os dados referentes ao estadiamento TNM. Vinte pacientes não apresentavam dados suficientes para o estadiamento completo. Dezessete por terem sido submetidos à ressecção local e 11 por apresentarem tumor irressecável, dentre estes 3 sem evidência de metástases à distância.

A média da contagem plaquetária foi $317.000 /$ ìl entre os pacientes com câncer e de 267.000/il entre os pacientes do grupo controle. $(p=0.006)$ A prevalência da trombocitose no câncer colorretal foi $32,1 \%$, sendo que $58,4 \%$ apresentavam um número de plaquetas acima da média do grupo controle.

Dentre os 108 óbitos ocorridos, 75 foram devido ao câncer. A prevalência de óbitos por câncer entre pacientes com trombocitose foi significativamente maior, de $56,7 \%$ contra somente $32 \%$ dentre os paci-

Tabela 1 - Características demográficas dos pacientes e localização do tumor.

\begin{tabular}{lcc}
\hline & $\mathbf{N}$ & $\mathbf{\%}$ \\
\hline Sexo & & \\
Masculino & 137 & 56,4 \\
Feminino & 106 & 43,6 \\
Localização do tumor & & \\
Reto & 124 & 51 \\
Sigmóide & 53 & 21,8 \\
Cólon descendente & 11 & 4,5 \\
Cólon tranverso & 13 & 5,3 \\
Cólon ascendente & 25 & 10,3 \\
Ceco & 17 & 7 \\
\hline
\end{tabular}


Tabela 2 - Estadiamento TNM.

\begin{tabular}{lrc}
\hline & $\mathbf{N}$ & $\mathbf{\%}$ \\
\hline Tumor & 12 & 5 \\
Tis & 15 & 6,3 \\
T1 & 34 & 14,2 \\
T2 & 141 & 59 \\
T3 & 37 & 15,5 \\
T4 & & \\
Invasão Linfonodal & 127 & 59,1 \\
Ausente & 88 & 40,9 \\
Presente & & \\
Metástase & 202 & 83,1 \\
Ausente & 41 & 16,9 \\
Presente & & \\
TNM & 1 & 0,4 \\
0 & 33 & 14,7 \\
I & 81 & 36,3 \\
II & 67 & 30 \\
III & 41 & 18,6 \\
IV & &
\end{tabular}

entes com contagem plaquetária normal. (Figura 1) Esta diferença foi ainda mais significativa quando se utilizou a média plaquetária dos controles, ou seja, entre o grupo 1 e 2. (Figura 2)

A média da contagem plaquetária foi de 356.729/il entre os óbitos e de 289.680/il entre os pacientes que não morreram $(\mathrm{p}=0.0001)$.

Dentre os 170 pacientes com seguimento no hospital, cinqüienta e três $(30,4 \%)$ tiveram recidiva da doença. O local predominante foi o fígado em $39 \%$ dos casos, seguido da recidiva local em $26 \%$.

A recidiva ocorreu com maior frequiência dentre os pacientes com trombocitose. Desses, dezessete $(36,2 \%)$ de um total de 47 tiveram recidiva da doença, contra 36 dos 126 pacientes com contagem de plaquetas normal $(29,3 \%)$. Essa diferença não foi significativa com p 0,38. (Figura 3)

Já quando se utilizou a média do grupo controle, o grupo 2 apresentou 36 de 90 pacientes (40\%) com recidiva tumoral, enquanto no grupo 1 isto ocorreu em apenas 12 de $67(17,9 \%)$. Sendo esta diferença bastante significativa. (Figura 4)

A média da contagem de plaquetas entre aqueles que tiveram recidiva da doença foi de $322.313 / \mu l$. No grupo que não apresentou recidiva esta média foi

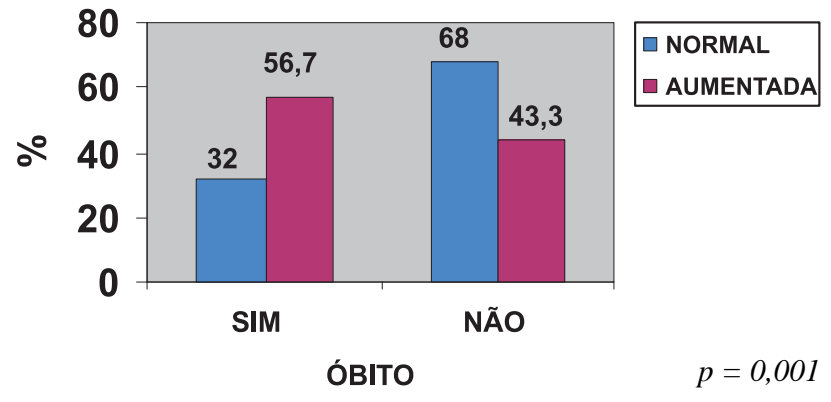

Figura 1 - Comparação entre a contagem plaquetária no préoperatório e óbitos devido ao câncer.

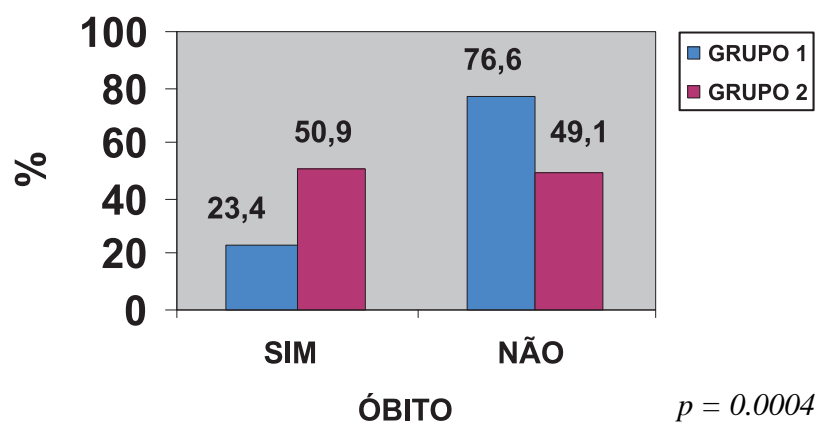

Figura 2 - Comparação entre os grupos 1 e 2 com relação aos óbitos devido ao câncer.

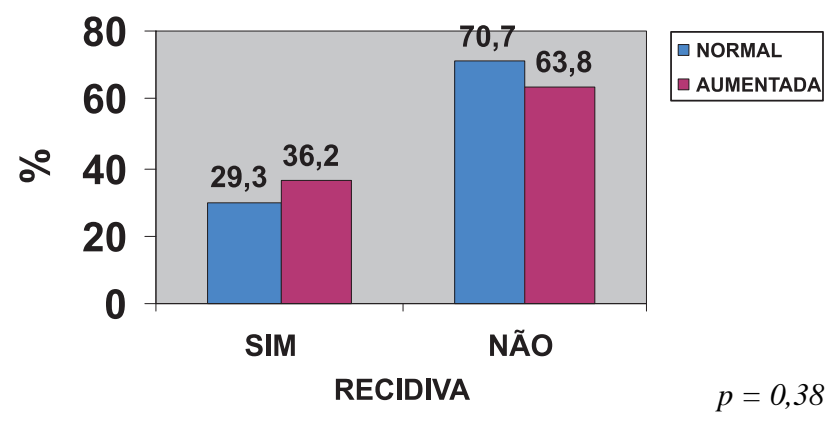

Figura 3 - Comparação entre a contagem plaquetária no préoperatório e recidiva da doença.

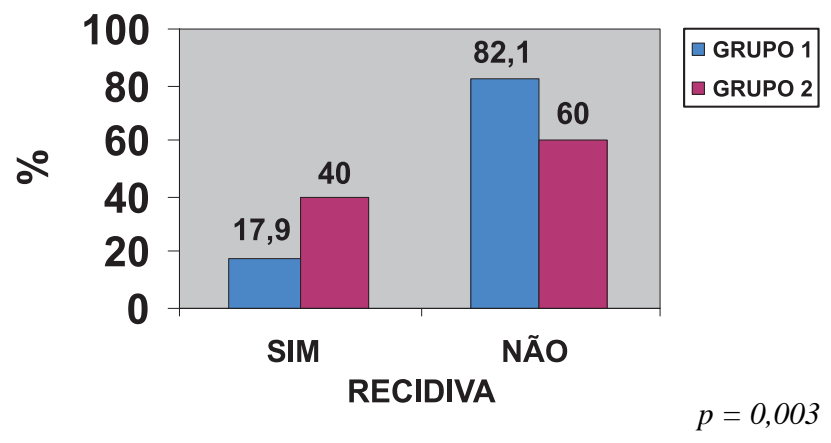

Figura 4 - Comparação entre os grupos 1 e 2 com relação à recidiva tumoral. 
de $297.027 / \mu 1$. Esta diferença também foi estatisticamente significativa com $\mathrm{p}$ de 0,02 .

Quanto ao estadiamento TNM, houve um maior número de pacientes no grupo com trombocitose com estadios mais avançados, 18/74 (32,5\%) apresentavam estadio IV, e apenas $7 / 74(9,4 \%)$ estadio I. No grupo em que a contagem de plaquetas foi normal houve um predomínio dos estádios mais precoces, 23/149 $(15,4 \%)$ estadio IV e $26 / 149(17,5 \%)$ estadio I. Não houve diferença estatística como mostra a figura 5.

Já quando se comparou os grupos 1 e 2 houve diferença significativa como mostra a figura 6 . No grupo $1,18 / 73(24,6 \%)$ eram estadio I e $8 / 73(11 \%)$ estadio IV. No grupo II, $13 / 136(9,6 \%)$ eram estadio I e $31 / 136(22,8 \%)$ estadio IV.

$\mathrm{Na}$ comparação das médias da contagem plaquetária em cada estadio houve diferença significativa. As médias foram maiores quanto maior foi o estadio do tumor.

Quanto à infiltração tumoral (T), também houve um maior número de pacientes do grupo que apresentaram contagem de plaquetas normal no estadio T1. Um total de $14 / 163$ pacientes $(8,6 \%)$ do grupo com estadiamento T1 e 13/163 (8\%) com estadiamento T4. Já no grupo com plaquetas aumentadas, somente um paciente de um total de $76(1,3 \%)$ apresentavam estadiamento T1 e 24/76 (31,6\%) estadio T4. Essa diferença foi significativa com $\mathrm{p}$ de 0,00001. (Figura 7)

Quando se utilizou a média da contagem plaquetária dos controles, notou-se o mesmo padrão que também foi estatisticamente significante, ou seja, no grupo 1 houve uma predominância do estadio T1 e T2, doze de um total de $85(14,1 \%)$ e $15 / 85(17,6 \%)$ respectivamente. Já no grupo 2 , houve predomínio dos pacientes com estadio T3 e T4, oitenta e oito pacientes de um total de $138(63,8 \%)$ e 27/138 (19,5\%) respectivamente. (Figura 8)

Comparando-se as médias plaquetária, os pacientes T1 apresentaram média de 241.400/1l, T2 de 278.161/1l, T3 de 315.157/il e T4 de 411.719/11. O valor de $\mathrm{p}$ foi de 0,00002 .

Quanto à invasão linfonodal, no grupo sem trombocitose 87 pacientes de um total de $146(59,6 \%)$ não apresentavam comprometimento de linfonodos (N0), em 28/146 (19,2\%) havia envolvimento N1 e em $31 / 146(21,2 \%)$ N2. No grupo com trombocitose em 40 de 69 pacientes (58\%) não havia comprometimento dos linfonodos, em 16/69 (23,2\%) havia envolvimento

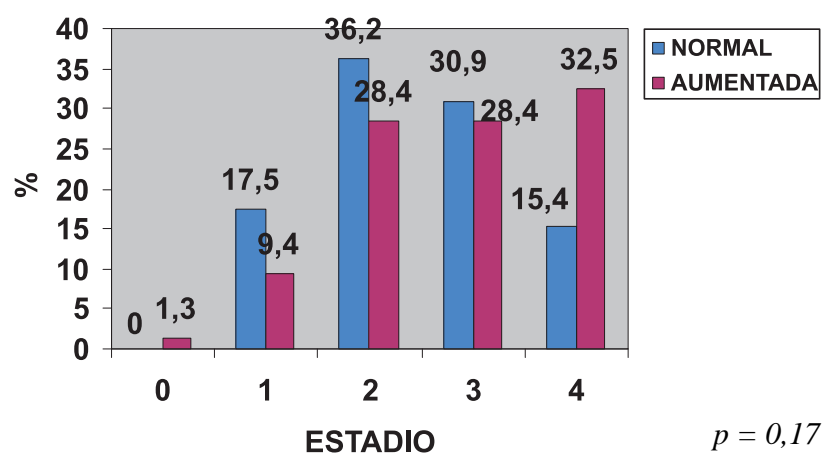

Figura 5 - Comparação entre a contagem plaquetária no préoperatório e estadiamento TNM.

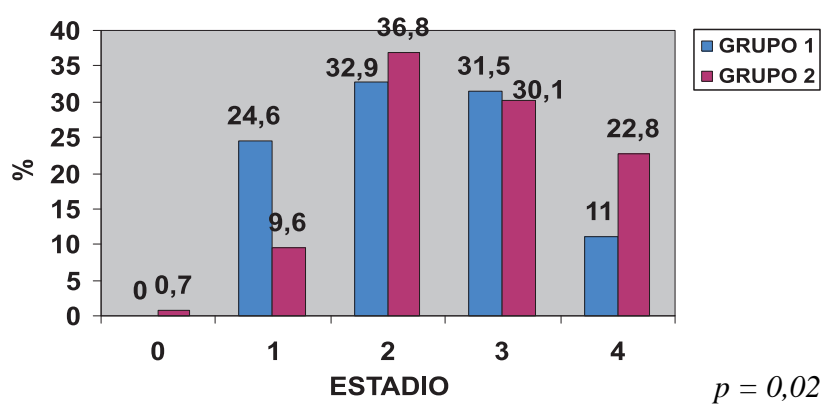

Figura 6 - Comparação entre os grupos 1 e 2 com relação ao estadiamento TNM.

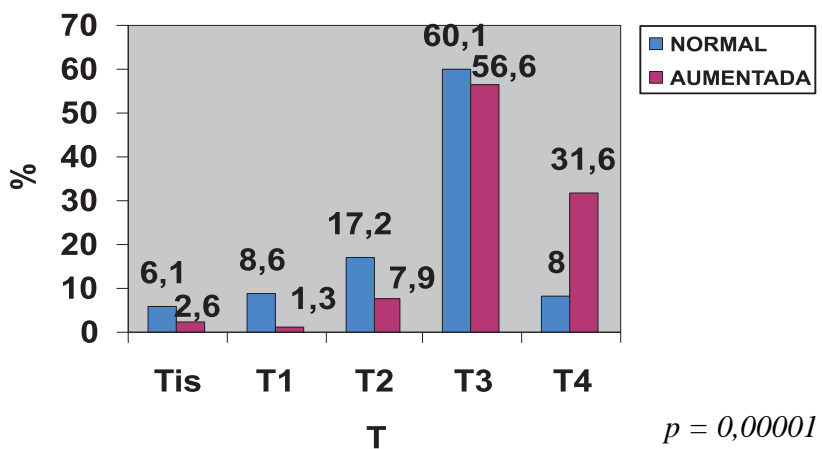

Figura 7 - Comparação entre a contagem plaquetária no préoperatório e infiltração tumoral $(T)$.

N1 e em 13/69 $(18,8 \%)$ N2. Não houve diferença estatística $(\mathrm{p}=0,77)$.

Quando se levou em conta a média dos controles, no grupo 1, 43 de 73 pacientes (59\%) não tinham invasão de linfonodos, em 15/73 (20,5\%) havia comprometimento N1 e em 15/73 (20,5\%) N2. No grupo 2, 74 de 128 pacientes $(57,8 \%)$ não tinham linfonodos comprometidos, 27/128 $(21,1 \%)$ eram N1 e $27 / 128(21,1 \%)$ eram $\mathrm{N} 2$. O valor de $\mathrm{p}$ foi de 0,98 . 
Também não houve diferença estatística entre as média dos três níveis de envolvimento linfonodal, que foram semelhantes $(\mathrm{p}=0,47)$.

A presença de metástases a distância foi encontrada em 23 de um total de 165 pacientes $(13,9 \%)$ do grupo com plaquetas normais e em $18 / 78$ pacientes $(23,1 \%)$ do grupo com trombocitose confome mostra a figura $9(\mathrm{p}=0,07)$.

Já quando se utilizou a média do grupo controle, as metástases foram encontradas em $8 / 85$ pacientes $(9,4 \%)$ do grupo 1 e em $31 / 142(21,8 \%)$ do grupo 2 conforme a figura $10(\mathrm{p}=0,02)$.

As médias da contagem plaquetária foram significativamente maiores dentre os pacientes com metástases com p de 0,02. A média dos pacientes

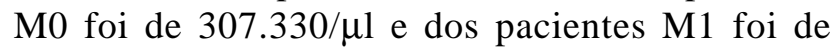
$368.162 / \mu 1$.

\section{DISCUSSÃO}

Nesse estudo, a prevalência da trombocitose foi de $32 \%$ dentre os pacientes com câncer colorretal. Dado semelhante ao que foi constatado por Constantini et $a l^{13}$, que encontrou uma prevalência de $33 \%$.

Houve uma associação significativa do número aumentado de plaquetas com óbitos por câncer, recidiva tumoral, infiltração tumoral, presença de metástases e estadiamentos mais avançados, principalmente quando se comparou ao grupo controle.

Isto já não foi evidenciado quando se comparou a presença de envolvimento linfonodal. Provavelmente devido às várias limitações deste estudo, principalmente o fato de ser retrospectivo e apresentar uma amostragem pequena, impedindo a análise isolada de cada uma das variáveis. Como a nossa amostra de pacientes foi pequena não foi possível avaliar separadamente o prognóstico dos pacientes com envolvimento linfonodal e trombocitose. Esses pacientes talvez apresentem um maior número de óbitos e recidivas do que aqueles com contagem plaquetária normal. Kandemir et $a l^{14}$ avaliou 198 pacientes com câncer colorretal, os quais não apresentavam envolvimento linfonodal, e encontrou uma prevalência de $12,1 \%$ de trombocitose e uma menor sobrevida dentre estes pacientes. Também constatou associação da trombocitose com tumores pouco diferenciados e infiltração tumoral.

Cravioto et al ${ }^{15}$ estudou 176 pacientes com câncer no reto e evidenciou um aumento do risco de

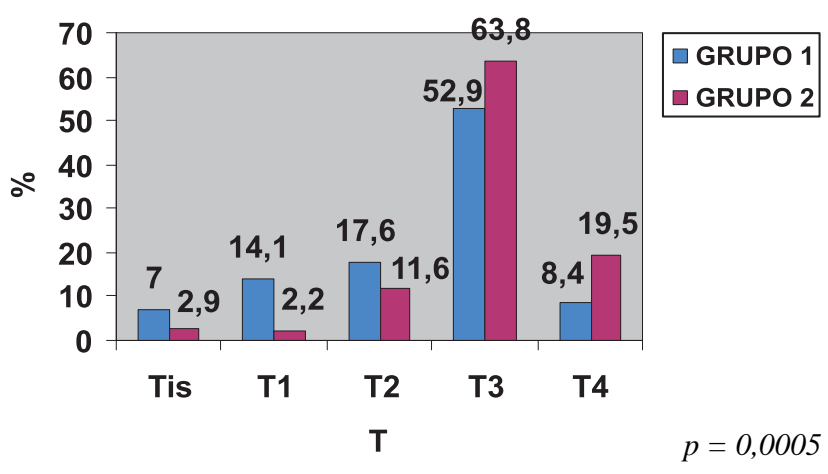

Figura 8 - Comparação entre os grupos 1 e 2 com relação à infiltração tumoral (T).

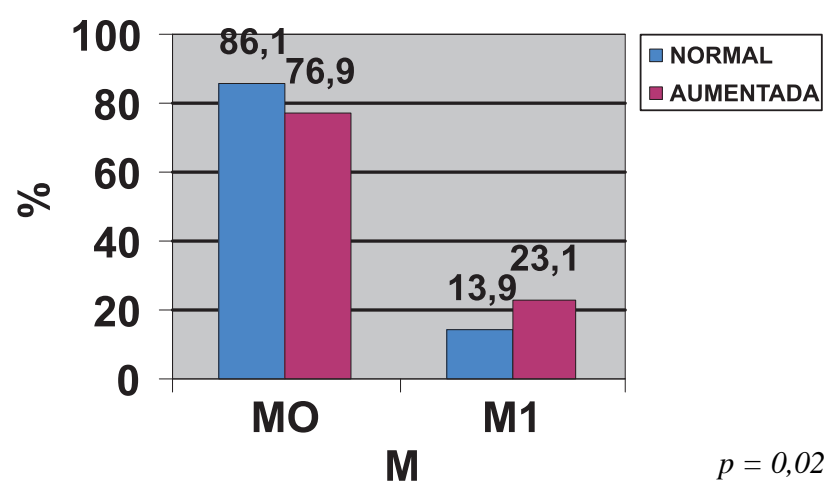

Figura 9 - Comparação entre a contagem plaquetária no préoperatório e presença de metástases.

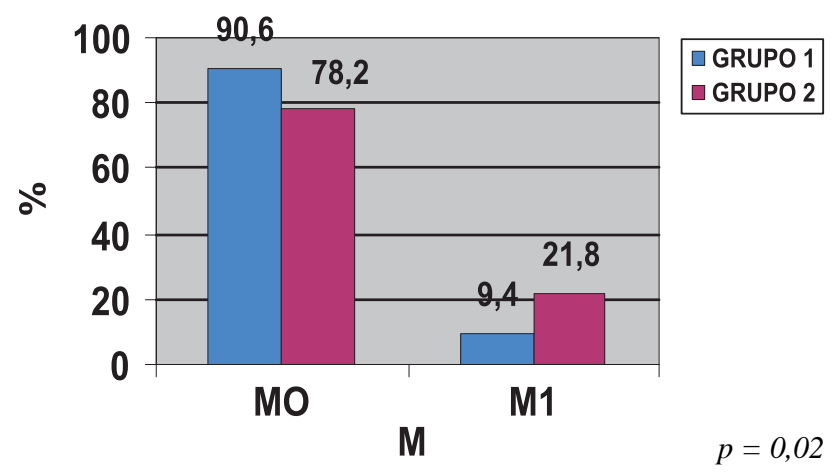

Figura 10 - Comparação entre os grupos 1 e 2 com relação presença de metástastes à distância (M).

doença metastática entre pacientes com contagem de plaquetas aumentada. Monreal et al ${ }^{16}$ também encontrou uma menor sobrevida para paciente com trombocitose e câncer colorretal.

Embora o mecanismo específico pelo qual a trombocitose atua nas doenças malignas ainda seja desconhecido, existem várias hipóteses. Uma delas é a produção de citocinas pela medula óssea em respos- 
ta ao crescimento tumoral. A interleucina- 6 parece um potente estimulador da megacariocitopoiese ${ }^{17}$.

A trombocitose pode afetar a sobrevida dos pacientes com câncer por afetar a invasão celular e as metástases. Há evidência de que as plaquetas protegem as células tumorais do sistema imune do hospedeiro $^{18}$. As plaquetas também facilitam a adesão de células tumorais ao endotélio vascular devido a formação de um trombo tumoral e interação com proteínas plaquetárias ${ }^{19}$.

Após a adesão, as plaquetas podem promover a proliferação de células tumorais através da secreção de agentes como o fator de crescimento endotelial vascular (VEGF). As plaquetas aderidas às células tumorais circulantes podem liberar VEGF nos pontos de adesão do endotélio, promovendo o aumento da permeabilidade vascular, contribuindo para o extravasamento de células tumorais e formação de metástases. VEGF é um dos mais potentes fatores pró-angiogênicos, sua concentração é fator prognóstico em vários tipos de câncer. Pacientes com câncer apresentam concentrações altas de $\mathrm{VEGF}^{20}$.

As plaquetas também apresentam outros fatores de crescimento vascular, como o fator de crescimento endotelial derivado de plaquetas e a trombospondina. Ambos também promovem a progressão tumoral e formação de metástases ${ }^{21,22}$.

\section{CONCLUSÃO}

A contagem de plaquetas no pré-operatório parece ser útil em identificar pacientes com prognóstico desfavorável, principalmente devido ao maior número óbitos por câncer e recidivas observados. Esses pacientes também apresentam estádios mais avançados no momento do diagnóstico. Mas, estudos prospectivo e com maior número de pacientes ainda são necessários para comprovar esta associação.

ABSTRACT: Aims - To investigate the prognostic value of thrombocytosis in patients with colorectal cancer. Method - Charts of 243 patients with colorectal cancer that had undergone surgical treatment were retrospectively reviewed. Data on TNM staging, tumor recurrence and cancer death were compared with the occurrence of thombocytosis before surgery. The control group was formed by fifty patients submitted to herniorrhaphy. Patients were divided into two groups on the basis of mean platelet count of control group: group 1, patients with platelet count below that mean and group 2, patients with platelet count above that level. Results - The mean platelet count was 317000/il among cancer patients and 267000/il in the control group patients. Prevalence of thrombocytosis in patients with colorectal cancer was $32,1 \%$. The cancer death rate was $56,7 \%$ in patients with thrombocytosis and $32 \%$ in patients with normal platelet count $(p=0,001)$. When the platelet count mean of control group was used, the difference was significantly greater ( $p=0004)$. Tumor recurrence occurred in $40 \%$ of group 2 patients and in 17,9\% of group 1 patients ( $p=0,003$ ). Regarding $\mathrm{T}$ staging, on group $1,14,1 \%$ of patients were $\mathrm{T} 1$ and $8,4 \%$ were $\mathrm{T} 4$. On group $2,2,2 \%$ were $\mathrm{T} 1$ and $19,5 \%$ were T4 (p=0,0005). Distant metastasis were found in 9,4\% patients of group 1 and $21,8 \%$ of group 2 (p=0,02). Considering TNM staging, group 1 had $24,6 \%$ of patients in stage 1 and $11 \%$ in stage 4. On group 2,9,6\% belonged to stage 1 and $22,8 \%$ to stage 4 $(p=0,02)$. Conclusion - Preoperative platelet count seems to be a helpful indicator of patients with poor prognosis.

Key words: Thrombocytosis; colorectal cancer; prognosis; pratelet.

\section{REFERÊNCIAS}

1. O'Brien MJ. Cancer of the colon and rectum: current concepts of aetiology and pathogenesis. Ir J Med Sci 1988;157:5-15.

2. Ratto C, Sofo L, Ippoliti M, Merico M, Doglietto GB, Crucitti F. Prognostic factors in colorectal cancer. Dis Colon Rectum 1999; 41: 1033 - 1049 .

3. Midgley R, Kerr D. Colorectal cancer. Lancet 1999; 353: 391 $-399$.

4. Liefers GJ, Cleton-Jansen AM, van de Velde CJ, Hermans J, van Krieken JHJM, Cornelisse CJ, et al. Micrometastases and survival in stage II colorectal cancer. N Engl J Med 1998; 339: $223-228$.
5. Riess L: Zur pathologischen anaotmie des blutes. Arch Anat Physiol Wissensch Med 1872; 39: 237 - 249.

6. Levin J, Conley CL. Thrombocytosis associated with malignant disease. Arch Intern Med 1964; 114: 497 - 500.

7. Engan T, Hanisdal E. Blood Analysis as prognostic factors in primary lung cancer. Acta Oncol 1990; 29: 151-4.

8. Pedersen LM, Milman N. Prognostic significance of thrombocytosis in patients with primary lung cancer. Eur Respir J 1996; 9: 1826-30.

9. Ikeda M, Furukawa H, Imamura H, Shimizu J, Ishida H, et al. Poor prognosis associated with thrombocytosis in patients with gastric câncer. Ann Surg Oncol 2002; 9(3): 287-291. 
10. Symbas NP, Townsend MF, El GR, Keane TE, Graham SD, Petros JA. Poor prognosis associated with thrombocytosis in patients with renal cell carcinoma. BJU Int 2000; 86: 203-7.

11. Rodriguez GC, Clarke PD, Soper JT, Berchuck A, Synan I, Dodge RK. The negative prognostic implications of thrombocytosis in women with stage IB cervical cancer. Obstet Gynecol 1994; 83: 445-8.

12. Hernandez E, Donohue KA, Anderson LL, Heller PB, Stehman FB. The significance of thrombocytosis in patients with locally advanced cervical carcinoma: a Gynecologic Oncology Group stydy. Am J Obstet Gynecol 1994; 170:549-54.

13. Costantini V, Zacharski LR, Moritz TE, Edwards RL. The platelet count in carcinoma of the lung and colon. Thromb Haemost 1990; 64:501 - 505.

14. Kandemir EG, Mayadagli A, Karagoz B et al. Prognostic significance of thrombocytosis in node-negative colon cancer. J Int Med Res 2005; 33(2):228-35.

15. Cravioto A, Luna-Perez P, Rodriguez S, Gutierrez M, Labastida $\mathrm{S}$. Thrombocytosis as a prognostic factor of survival in patients with rectal câncer. Ann Surg Oncol 2004; 11(2): S118.

16. Monreal M, Fernandez-Llamazares J, Pinol M, Julian JF, Broggi M, Escola D, et al. Platelet count and survival in patients with colorectal cancer - a preliminary study. Thromb Haemost 1998; 79: 916 - 918 .
17. Lidor YJ, Xu FJ, Martinez MO, et al. Constitutive production of macrophage colony-stimulating factor and interleukin- 6 by human ovarian surface epithelial cells. Exp Cell Res 1993; 207: 332-9.

18. Nakazi H. Preoperative and postoperative cytokines in patients with cancer. Cancer 1992; 70: 709-13.

19. Kin YJ, Borsig L, Varki NM, Varki A. P-selectin deficiency attenuates tumor growth and metastasis. Proc Natl Acad Sci USA 1998; 95: 9325-30.

20. Nash GF, Turner LF, Scully MF, Kakkar AK. Platelets and cancer. Lancet Oncol 2002; 3: 425-30.

21. Takebayashi R, Akiyama S, Akiba S, Yamada K, Miyedara K, Sumizawa $\mathrm{T}$, et al. Clinicopathologic and prognostic significance of an angiogenic factor, thymidine phosphorylase, in human colorectal carcinoma. J Natl Cancer Inst 1996; 88: $1110-1117$.

22. Qian X, Tuszynski GP. Expression of thrombospondin-1 in cancer: a role in tumor progression. Proc Soc Exp Biol Med 1996; 212: 199 - 207.

\section{Endereço para correspondência:} RENATO DE ARAÚJO BONARDI

Rua Olavo Bilac, 680

CEP 80440-040 - Curitiba-PR

Fone: (41) 3244-8963 - (41) 3232-1262

Fax: (41) 3243-8827

E-mail: rbonardi@onda.com.br 